\title{
Survey Paper on loT based Smart Intelligent Toilet using SVM and Regression Algorithms
}

\author{
Vasudha K. \\ Global Academy of Technology \\ Bangalore \\ India
}

\author{
Yashaswini S. \\ Global Academy of Technology \\ Bangalore \\ India
}

\author{
Ashwini K, PhD \\ Global Academy of Technology \\ Bangalore \\ India
}

\begin{abstract}
This paper proposes the concept of Machine Learning and Internet of Things (IoT) to implement a Smart Intelligent Toilet. Machine learning is used to regulate the formation of crucial models in order to enable algorithms to learn with the help of available data. IoT is regarding embedding system to the internet. Machine learning and IoT has experienced a boost in acceptance among many fields including the medical field. The modern and upgraded laboratory is very much necessary as diagnosis of a disease and analysis of a person's health in a precise form is very much important. Artificial intelligence creates a platform to be precise in the measurement of any parameters using various algorithms. In this paper, we propose to apply Regression algorithm to predict the output values like urine test, based on input features such as urine sample from data sets fed in the system. The algorithm builds a model based on the features of the training dataset and also makes use of the model to predict value for new data. Support Vector Machine(SVM) is a machine learning algorithm used for both classification and regression challenges. IoT devices fail to function without artificial intelligence and artificial intelligence in turn needs IoT devices to be of better use for a smarter human kind. Both these technologies jointly hold the power to alter our lives to better standards.
\end{abstract}

\section{General Terms}

Pattern Recognition, Security, Machine Learning Algorithms, Internet of Things (IoT), Artificial Intelligence.

\section{Keywords}

SVM, regression, urine analyzer, Global System for Mobile(GSM), electrocardiogram, bio- impedance strategy , Raspberry pi microprocessor, aurdino board.

\section{INTRODUCTION}

Machine learning is when a computer has been taught to distinguish patterns by providing it with data. And algorithms to help comprehend that data. We call the process of learning as 'training' and the output this process produces is called a 'model'. A model can be given new data and it can reason about this new information based on what it has formerly learned. Machine learning models identify a set of rules using huge amount of computing power that human brain will not be able to process. The more data a machine learning model is fed, the more intricate the rules and the more accurate the predictions [1].

Three types of models are used in machine learning classification, clustering and regression. In the proposed system we concentrate more on Regression used in medical field. Regression is a Machine learning technique which can be used to examine report of a person and predict the health status. Applications of AI in medical diagnostics are in the early adoption phase across multi-specialty with limited data currently available on patient outcomes. These applications can influence how doctors and health care systems approach diagnostics and how an individual can understand changes in their health in real time.

Internet of Things is on the edge to transform how we keep people safe and healthy especially as the requirement for a solution to reduce healthcare costs increase in the coming years. IoT can help monitor, inform and notify not only care givers, but provide physicians with original data to identify issues before they become serious [2].

Machine learning concepts combined with IoT can be implemented instead of performing manual diagnosis of a disease for example, by performing urine analysis in labs. Urine analysis is mainly conducted for the diagnosis of renal disorders. Urine analysis is used in chemical medicine diagnosis method. Urine analysis is used to test some chemical composition in the urine. It uses original urine as a urine sample for processing and testing. The analysis quantitatively or semi quantitatively tests the level of bilirubin, ketones, protein, glucose in the urine according to some changes in the colors of the urine sample. The simple way of obtaining urine specimens and lesser cost of test makes urine analysis as an important part of diagnosis. The usage of machine learning classifiers such as Support Vector Machine (SVM) can be implemented for the purpose of urine analysis. SVM algorithms can be used to deal with the problem of imbalanced data in urine analysis by employing efficient monitoring [3].

From monitoring and diagnostics to delivery methods, IoT sensors in health care combine sensor output and communications to provide functions that were considered theoretical. With advancements of IoT sensors in healthcare, the industry now includes a varied collection of patient data to support diagnostics and preventive care and even measure the possible outcome of the preventive treatment [4].

Usage of IoT sensors are widespread but for diagnostic purposes, the IoT sensors can be embedded into devices used by the patient in his daily life, for example, monitoring of 
heart function on his chair. While measurements in a bed or on a chair always have to deal with the clothing between the sensors and human body, measurement on toilet seat provides direct skin contact to the sensors and also enable urine analysis. The proposed system is expected to do all the monitoring and analysis of the person when he comes in contact with the sensors.

\section{EXISTING SYSTEM}

So far, enormous amount of work has been done to build a Smart Intelligent Toilet System using IoT. This section describes the work carried out so far.

Laksmi Kadlimatti has proposed the work which describes about analyzing urine to know a person's health information. Urine Analyzer is used for quantitative analysis of urine in real time i.e. analyzing contents of urine. The system contains analytical chamber, automation unit, bio chemical sensors to determine amount of contents in urine. It also uses microcontroller for signal processing and GSM based data transmitter. This hardware is used to transmit the values obtained to a doctor. The method used here is optical detection for classification algorithm implemented using neural network. It uses RGB parameters which it acquires using color sensors. This type of system is mainly used to treat diabetes and heart failure. The advantages of this paper are that practical implementation is simpler and it proposes innovative, intelligent and hassle-free confidential way of urine analysis. The disadvantage is that it is financially not viable and initial cost of design and installation is costlier [5]

.Ki Tae. Nam has proposed the work which focuses on ubiquitous healthcare which means monitoring a person's health around his/her own living place that is, they can avoid visiting labs in order to get them checked. A bio-signal detector is attached to the lavatory and an analogue circuit to process the bio signal. The results are displayed on an LCD board and also sent to doctors via Email and Bluetooth. Electrodes are affixed to the seat. Here also microcontrollers are used. The method employed is linear system identification which is used for signal processing and provides means to send the result to a doctor. This type of system is mainly used to treat cardiovascular issues. The advantages of this paper are all processes are done automatically and it emphasizes on ubiquitous health care technology. The main disadvantage of this paper is the method for evaluating data is not adequate[8].

T.Schlebusch (2011) has proposed the work which describes embedding measuring devices in daily life objects like the toilet seat. His work convinces us that toilet seat is the best to measure one's health because other object such as a chair includes clothing in between. Hence, it is called unobtrusive

i.e. nothing in between. It takes electrocardiogram and bioimpedance strategy and shows the practicality of measuring them on toilet seat. It uses weight sensors and results are transmitted to CCU and stored in a database via UMTS repeater. This paper says that intelligent toilet has high potential to increase quality of medical care at home. The advantages of this paper are that the system developed performs a comprehensive health check and such a system has a high possibility to boost the quality of medical care. Direct skin contact to sensors makes it more efficient. The disadvantage of this paper is that electrodes are disposable [9].

Table 1. Comparison of existing systems

\begin{tabular}{|cc|c|c|c|}
\hline Author & Methodology & Advantages & Disadvantages \\
\hline $1 . \quad$ Laksmi Kadlimatti & $\begin{array}{c}\text { Optical detection of various } \\
\text { colours on the urine test } \\
\text { strips. }\end{array}$ & $\begin{array}{c}\text { 1. Innovative, } \\
\text { intelligent and hassle-free } \\
\text { confidential way of urine } \\
\text { analysis. }\end{array}$ & $\begin{array}{c}\text { Initial cost of design and } \\
\text { installation is costly. }\end{array}$ \\
\hline $2 . \quad$ Ki Tae. Nam & $\begin{array}{c}\text { Linear parametric system } \\
\text { identificat-ion method. }\end{array}$ & $1 . \quad \begin{array}{c}\text { Ubiquitous health care } \\
\text { technology. }\end{array}$ & $\begin{array}{c}\text { Method to evaluate data not } \\
\text { adequate. }\end{array}$ \\
\hline $3 . \quad$ T.Schlebusch & $\begin{array}{c}\text { Electro cardiogram and bioimped- } \\
\text { ance spectroscopy } \\
\text { measurements. }\end{array}$ & $1 . \quad \begin{array}{c}\text { Direct skin contact to } \\
\text { sensors. }\end{array}$ & $1 . \quad$ Electrodes are disposable. \\
\hline
\end{tabular}




\section{PROPOSED SYSTEM}

$\mathrm{pH}$ sensor can be used to measure the $\mathrm{pH}$ parameter of a patient's urine along with aurdino board and Raspberry Pi microprocessor. $\mathrm{pH}$ of urine gives us a lot of information about the health status of a person. A neutral $\mathrm{pH}$ is 7.0. A larger number indicates it is more basic (alkaline). A lower number indicates that the urine is more acidic. The average urine sample tests at about 6.0.If the urine sample is lower, this could indicate an environment conducive to kidney stones. Acidosis, dehydration ,diabetic, ketoacidosis ,diarrhea and starvation also prefer an acidic environment A higher- than-normal urine $\mathrm{pH}$ could indicate gastric suctioning that takes away stomach acids, kidney failure, kidney tubular

acidosis, pyloric obstruction, respiratory alkalosis, urinary tract infection, vomiting [10].

Given below is the sequence in which the proposed system will be implemented:

1) When the urine sample is fed to the sensor, the sensor produces data for further processing. Once the data is collected from the sensors, machine learning algorithms are used to compare the new data with the data already present in the system's database.

2) If the data patterns match, the disease is predicted directly using machine learning algorithms. The predicted result is displayed as the output.

3) If the data patterns don't match, we use further algorithms to find the most nearest match and then predict the disease.

For pattern-matching and prediction of diseases, we use the following machine learning algorithms:

1) Regression algorithm predicts the output values based on input features from data fed in the system, thego-to methodology is that the algorithm builds a model on the features of training data and using the model to predict value for new data[11].

2) Support Vector Machine(SVM) is a machine learning algorithm which can be used for both classification and regression challenges. Tuning parameters value for SVM improves the model performance[12].

Here we are trying to create a platform with efficient measurement system for different parameters, to avoid human errors by using machine learning algorithms.

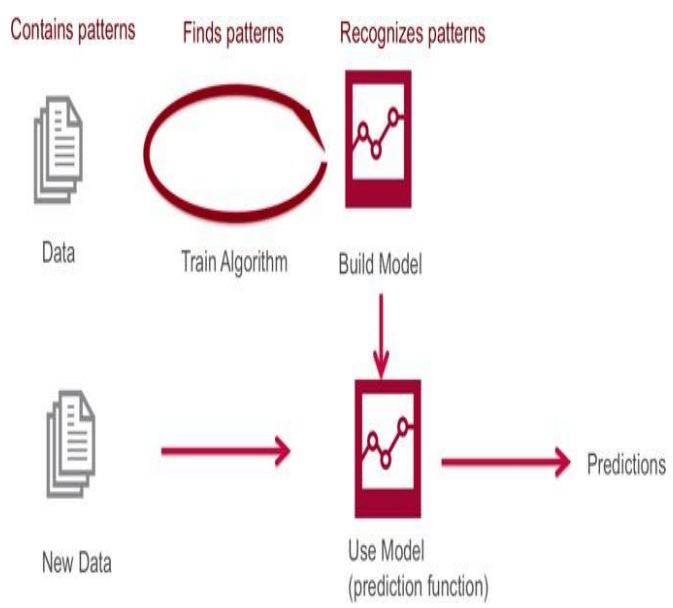

Figure 1: Proposed Prediction model

\section{CONCLUSION}

Modern era of science and technology is always trying to increase the comfort and improve the health conditions of humans. The machine learning and IoT developments in medical industry have intense impact on better living standards. We should analyze the benefits and need of regular health checkups and tests and develop an IoT solution architecture for example, an architecture to build a smart intelligent toilet. By making use of smart toilet, a user can get to know about a lot of medical conditions without even visiting a doctor or a lab. For such a smart toilet, the regression and SVM algorithms under machine learning can be used to develop a system and the IoT sensors can be used for the working of the system.

\section{REFERENCES}

[1] https://www.expertsystem.com/machine-learningdefinition/

[2] https://www.wipro.com/en-IN/business-process/what-caniot-do-for-healthcare-/

[3] https://www.surgeryencyclopedia.com/St$\mathrm{Wr} /$ Urinalysis.html

[4] https://www.sensorsmag.com/components/iot-sensorsthrive-healthcare-markets

[5] Laksmi Kadlimatti, Jyothi Hiremath, K Bhat "Design and development of automatic urine analyzer suitable for public toilets and transmission of vital composition to user's registered cell phone", International Research Journal of Engineering and Technology (IRJET).

[6] Sabeel T.M.A, CheHarun F.K., Eluwa S.E., Sabeel S.M.A, "Detection of volatile compounds in urine using an electronic nose instrument", Computing, Electrical and Electronics Engineering (ICCEEE)

[7] http://istep.ifmefector.com/2013/04/09/intelligent-toiletmonitors-your-health/

[8] Ki Tae. Nam, Jun Seob. Ko, Sung Bin. Park, and Hyung Ro. Yoon "The lavatory-typed health monitoring system with linear system identification scheme for home healthcare"Proceedings of the 29th Annual International Conference of the IEEE EMBSCité Internationale, Lyon, France.

[9] T.Schlebusch "Unobtrusive Health Screening on an Intelligent toilet seat".

[10] https://www.healthline.com/health/urine-ph

[11] https://www.analyticsindiamag.com/top-6-regressionalgorithms-used-data-mining-applications-industry/

[12] https://www.analyticsvidhya.com/blog/2017/09/understai ng-support-vector-machine-example-code/

[13] Carsten Röcker RWTH Aachen University, Germany Martina Ziefle RWTH Aachen University, Germany, "EHealth, Assistive Technologies and Applications for Assisted Living: Challenges and Solutions", MEDICAL INFORMATION SCIENCE REFERENCE Hershey • New York

[14] US patent on "Toilet device with health examination system", Patent No.: US 4961431 A.

[15] https://www.businesswire.com/news/home/20180828005 288/en/InnovativeApplications-IoT-Healthcare-InfinitiResearch 